\title{
Triple-negative breast lobular carcinoma: a luminal androgen receptor carcinoma with specific ESRRA mutations
}

\author{
Anthony Bergeron $\mathbb{D}^{1} \cdot$ Gaëtan MacGrogan $\mathbb{D}^{2,3} \cdot$ Aurélie Bertaut $^{4} \cdot$ Sylvain Ladoire ${ }^{5,6,7} \cdot$ Patrick Arveux $^{8}$. \\ Isabelle Desmoulins ${ }^{5}$. Hervé Bonnefoi ${ }^{3,9,10}$. Catherine Loustalot ${ }^{11}$. Sophie Auriol ${ }^{12}$. Françoise Beltjens ${ }^{1}$. \\ Emilie Degrolard-Courcet ${ }^{1} \cdot$ Céline Charon-Barra $^{1} \cdot$ Corentin Richard ${ }^{13} \cdot$ Romain Boidot $\mathbb{D}^{13} \cdot$ Laurent Arnould $\mathbb{D}^{1,6}$
}

Received: 3 September 2020 / Revised: 17 January 2021 / Accepted: 19 January 2021 / Published online: 22 March 2021

(c) The Author(s), under exclusive licence to United States \& Canadian Academy of Pathology 2021

\begin{abstract}
Primary triple-negative invasive lobular breast carcinomas (TN-ILCs), which do not express hormone receptors and HER2 at diagnosis, are rare and poorly known. In this study, we analyzed the largest TN-ILC series ever reported in the literature, in comparison to phenotypically similar breast tumor subtypes: triple-negative invasive ductal carcinoma (TN-IDC) and hormone receptor-positive invasive lobular carcinoma (HR + ILC). All primary TN-ILCs registered in our database between 2000 and 2018 ( $n=38$ ) were compared to tumors from control groups, matched by stage and Elston/Ellis grade, with regard to clinical, pathologic, and immunohistochemical characteristics. A comparative molecular analysis (whole-exome and RNA sequencing using next-generation technology) was also performed. We found that TN-ILC patients were older than those with HR + ILC $(P=0.002)$ or TN-IDC $(P<0.001)$. Morphologically, TN-ILCs had aggressive phenotypes, with more pleomorphism $(P=0.003)$ and higher nuclear grades than HR + ILCs $(P=0.009)$. Immunohistochemistry showed that TNILCs less frequently expressed basal markers (CK5/6, EGFR and SOX10) than TN-IDCs $(P<0.001)$, while androgen receptor $(\mathrm{AR})$ positivity was more prevalent $(P<0.001)$. Survival curves analysis did not show differences between TN-ILC and TN-IDC patients, while overall and distant metastasis-free survival were significantly worse compared to those with HR + ILCs $(P=0.047$ and $P=0.039$, respectively). At a molecular level, we found that TN-ILCs had particular transcriptomic profiles, characterized by increased AR signaling, and associated with frequent alterations in the PI3K network and ERBB2. Interestingly, whole-exome analysis also identified three specific recurrent ESRRA hotspot mutations in these tumors, which have never been described in breast cancer to date and which were absent in the other two tumor subtypes. Our findings highlight that TN-ILC is a unique aggressive breast cancer associated with elderly age, which belong to the luminal androgen receptor subtype as determined by immunohistochemistry and transcriptomic profiling. Moreover, it harbors specific molecular alterations (PI3K, ERBB2 and ESRRA) which may pave the way for new targeted therapeutic strategies.
\end{abstract}

Part of the data reported in this manuscript were presented as preliminary results (poster) at the 2020 USCAP Annual Meeting (Los Angeles, US).

Supplementary information The online version contains supplementary material available at https://doi.org/10.1038/s41379021-00742-9.

\section{Anthony Bergeron} abergeron@cgfl.fr

Extended author information available on the last page of the article

\section{Introduction}

Invasive lobular carcinoma (ILC) of the breast represents $5-15 \%$ of all breast cancers and is the second most frequent histologic subtype after invasive ductal carcinoma of no special type (IDC-NST) [1]. The classic ILC subtype is characterized by proliferation of non-cohesive small cells individually dispersed in fibrous connective tissue or organized in single-file linear cords invading the stroma. Its pleomorphic counterpart is a rare aggressive variant with more nuclear atypia and pleomorphism [1, 2]. ILCs often show the loss of E-cadherin expression coupled with $\mathrm{CDHl}$ alterations. Most of them express receptors for both steroid hormones: estrogen and progesterone (hormone receptor-positive tumors, $\mathrm{HR}+$ ), while HER2 amplification/overexpression is rare [1,3]. They usually 
have a low proliferation index and are often classified as luminal A by gene expression profiling [4]. Compared to IDCNST, ILCs develop in older patients. They are also bigger, of lower histologic grades, show less lymphovascular invasion as well as higher rates of bilateral and multifocal/multicentric disease and different responsiveness to therapy [5, 6]. Even though they have morphologic features associated with a good prognosis, they seem to give poor long-term outcomes, similar to those of IDC-NST, if not worse [3, 7, 8].

Triple-negative breast carcinomas (TNBCs), defined by the absence of hormone receptors (HR negativity) and of the HER2 expression [1], account for $10-17 \%$ of all breast cancers [9]. They are more frequent in younger women and in patients with BRCA mutations. These tumors are usually of higher grade and are associated with poor outcomes [9, 10]. Based on the intrinsic molecular subtyping of breast cancer, about $80 \%$ of them have a basal-like transcriptomic profile [11-13]. However, the results by Lehmann et al. [11] and others [12-14] suggested that TNBCs are more heterogeneous than previously described, and identified different molecular subtypes, each displaying a particular biology with molecular drivers that can be therapeutically targeted.

TNBCs have been the focus of many studies reported in the literature. However, most of these studies included mainly IDC-NSTs and not the triple-negative invasive lobular carcinomas (TN-ILCs), which are rare. Therefore, although some studies have reported the existence of rare TN-ILCs expressing basal cytokeratins [7, 15, 16], the data for TN-ILC remain scarce [17-20].

In order to fill this gap, in this study, we aimed to better characterize triple-negative invasive lobular breast carcinomas (TN-ILCs) by comparing their morphologic, immunophenotypic, molecular, and prognostic features to those of phenotypically close tumors: hormone receptors-positive invasive lobular carcinomas (HR + ILCs) and triple-negative invasive ductal carcinomas (TN-IDCs). Next-generation sequencing was used to search for specific genetic alterations and changes in the activation of specific signaling pathways.

\section{Materials and methods}

\section{Study design and samples}

The study group comprised all primary TN-ILCs $(n=38)$ diagnosed between January 2000 and December 2018 in the Georges-François Leclerc Cancer Center (CGFL, Dijon, France) and in the Bergonié Institute (Bordeaux, France). Patients with ILCs which were secondarily TN tumors (tumors which became TN at recurrence, following the treatment of initially non-TN primary breast tumors) were excluded.

Controls were 76 TN-IDCs and $76 \mathrm{HR}+$ ILCs, which were randomly selected from the database of the Côte d'Or
Breast Cancer Registry (France) and matched to cases by clinical stage and histologic grade.

Demographic and clinical characteristics of all subjects were retrieved from medical records. All samples were centralized in the CGFL before analysis and submitted to pathologic review. The latter included evaluating hematoxylin and eosin-stained (H\&E) slides from cases and controls, including both core biopsy and surgical resection slides when available. Only the most representative presystemic therapy section of the tumor was selected for histologic characterization and complementary analyses: surgical resection slides for patients who had not received neoadjuvant systemic therapy ( $n=31$ for TN-ILC, $n=45$ for TN-IDC, and $n=71$ for HR + ILC), and core biopsy slides for metastatic or non-operable patients ( $n=4$ for TNILC, $n=6$ for TN-IDC, and $n=1$ for HR + ILC), and for those who had received neoadjuvant chemotherapy before resection ( $n=3$ for TN-ILC, $n=25$ for TN-IDC, and $n=4$ for HR + ILC)). The TN status (both HRs and HER2 negativity) was confirmed in a retrospective review by two pathologists. For patients who underwent surgery before adjuvant systemic therapy, the tumor size was determined using the pT values, which had been defined according to the latest classification by the American Joint Committee on Cancer Staging (AJCC, 8th Edition) [21] and recorded in surgical pathology reports. For the others, the size was determined based on ultrasound scan reports. The multifocality was defined by the presence of at least two distinct tumors on macroscopic examination as reported in surgical pathology reports for patients who underwent primary surgery without neoadjuvant systemic therapy, and determined based on ultrasound scan reports for the others. The classic and pleomorphic ILC morphologic variants as well as the apocrine differentiation criteria (large cells with abundant eosinophilic granular cytoplasm, enlarged nuclei, and prominent nucleoli) were defined as in the Fifth Edition of the World Health Organization's Classification of Breast Tumors [1]. The three characteristics used in the E\&E grading system were re-scored by two pathologists on prechemotherapy samples for all subjects [22], and the TNM stage was defined using the latest classification by the AJCC (8th Edition) [21].

CGFL has been authorized to conduct scientific research by relevant French authorities (authorization number AC-2019-3531). Informed consent was obtained from all patients participating in the study and the study was approved by the CGFL Ethical and Scientific Committee.

\section{Immunohistochemistry}

Using immunohistochemical methods, we evaluated the expression of E-cadherin, an adhesion molecule whose loss is frequent in lobular breast carcinoma. Then, the expression 
of basal markers, both established (Cytokeratin 5/6 (CK5/6) and epidermal growth factor receptor (EGFR)) and recently suggested (SOX10), as well as of two potential prognostic and/or predictive markers (Androgen receptor (AR) and proliferation marker Ki67) was evaluated for all tumors. All antibody references and preparation details are summarized in Supplementary Table S1. The analyses were performed on $5 \mu \mathrm{m}$-FFPE tissue sections using an automated stainer (Ventana Benchmark XT system ${ }^{\circ}$, Roche ${ }^{\mathrm{TM}}$ ). The results were analyzed by two independent pathologists. In case of evaluation discrepancy, a consensus score was determined.

ER and PR markers were considered negative if less than $1 \%$ of tumor cells (TCs) were stained. Tumors containing 1-10\% HR-positive TCs (HR low tumors), which were defined in the latest World Health Organization's Classification of Breast Tumors as tumors with heterogeneous biological behaviors [1], were excluded from the study in order to avoid any bias. Only tumors with at least $10 \%$ of HR-positive TCs were included in the HR-positive-control group. HER2 staining was scored according to the ASCO/ CAP guidelines and considered positive when it showed 3 + HER2 staining or $2+$ HER2 staining with fluorescent in situ hybridization positivity [23]. E-cadherin staining was classified as negative when a loss of membranous expression in TCs was observed. Of note, TCs with aberrant cytoplasmic or Golgi type staining were defined as negative [24, 25]. Immunostaining for CK5/6, EGFR, and SOX10 were considered positive when $>1 \%$ of TCs showed a positive signal. AR was considered as positive if $\geq 10 \%$ of nuclear TCs were stained, as it is the commonly used cut-off $[26,27]$. For Ki67, the result was given by percentage of immunoreactive TCs.

\section{Molecular analysis}

Nucleic acids were extracted from FFPE specimens as described previously [28] and analyzed by whole-exome and RNA sequencing.

\section{DNA and RNA extraction}

Following the evaluation of the tumor cell content in FFPE tumor specimens by a pathologist, samples were macrodissected to obtain at least $80 \%$ tumor cell content for nucleic acid extraction. DNA was extracted using the Maxwell-16 FFPE Plus LEV DNA purification kit (Promega) according to the manufacturer's protocol. RNA was extracted from the same specimens using the Maxwell-16 LEV RNA FFPE Purification kit (Promega) according to the manufacturer's protocol. DNA and RNA quality were assessed by spectrophotometry with absorbance at 230, 260, and $280 \mathrm{~nm}$. DNA was quantified using the Qubit fluorometric assay (Life Technologies).

\section{Whole-exome sequencing}

Libraries were constructed from 200 ng DNA and captured using the SureSelect Human All Exon v6 kit (Agilent) following the manufacturer's protocol. Paired-end $(2 * 111$ bases) sequencing was performed on a NextSeq500 device (Illumina). Next, the sequences were aligned and annotated with the human $\mathrm{Hg} 19$ genome based on the SureSelect Human all Exon v6 manifest using the BWA and GATK algorithms. Only sequences with a read depth of $10 \times$, a mutant allele frequency above $5 \%$, and a frequency in the general population inferior to $1 \%$ were retained for further analyses.

Whole-exome analyses were performed as described previously [29]. VCF files were annotated with the VEP software [30]. Only variants with missense mutations or frameshifts on canonical transcript, with the prevalence below $0.05 \%$ in the general population and below $95 \%$ among our patients were retained. Out of 17,970 genes identified as altered, those reported to be usually altered in breast cancer [31,32] were compared between the three groups. Mutations were retained only if they had been previously described as pathogenic or likely pathogenic variants, and variants of unknown significance if predicted to be deleterious by both Sift and Polyphen software. Genes mutated specifically in TN-ILC were selected using the Fisher exact test with a $1 \%$ significance threshold. Overall, 177 altered genes were retained for further analysis.

\section{RNA sequencing}

rRNA-depleted RNA was used for the library preparation with the NEBNext Ultra II Directional RNA library prep kit for Illumina (New England Biolabs) according to the manufacturer's instructions. Libraries were paired-end sequenced ( $2 * 76$ base pairs) on a NextSeq500 device (Illumina), with a read depth of 20 million.

Kallisto software [33] was used for quantifying transcript abundance from RNA-seq data against GRCh38 cDNA reference transcriptome from the Ensembl database, v96. Only protein-coding transcripts and genes were included in the downstream analysis. Differential expression analysis was performed using the DESeq2 $\mathrm{R}$ package [34]. Genes with an absolute log fold-change greater than 1 and the $s$-value below 0.005 were considered as significantly differentially expressed. Pathway enrichment analysis was performed using the Enrichr website.

Intrinsic molecular subtype classification of tumors following RNA sequencing was conducted based on the PAM50 gene set [35] using the genefu R package version 2.22.0 (http://www.pmgenomics.ca/bhklab/software/genefu) as described elsewhere [36]. Lehmann's TBNC subtype classification was performed using the TNBC-type online 
tool (https://cbc.app.vumc.org/tnbc/index.php) as described in $[11,37]$. Both classifications were done on Transcripts Per Millions values.

\section{Statistical analysis}

Continuous variables were expressed as numbers of observations, means (with standard deviation) and medians (with min-max), and compared using Student or Wilcoxon tests as appropriate. Categorical variables, expressed as frequencies and percentages, were compared using the $\mathrm{Chi}^{2}$ or Fisher test. For survival analysis, survivors were censored at the date of last follow-up and distant metastasis-free survival was defined as the interval from diagnosis until distant recurrence date. The median follow-up was calculated using the reverse Kaplan-Meier method. Survival times and rates were determined using the Kaplan-Meier method and comparisons were performed with the log-rank test. Independent predictors of survival were determined using Cox regression univariate and multivariate analyses. Covariables with $P$ values below 0.200 in univariate analysis were included in the multivariate model, while variables with more than $20 \%$ missing data were excluded. Hazard ratios were determined with $95 \%$ confidence intervals. Tests were two-sided and the significance threshold was set at $5 \%$. All statistical analyses were performed using the SAS software, version 9.4.

\section{Results}

We analyzed clinical, histological, and molecular characteristics of 38 TN-ILC tumors in comparison to TN-IDCs and HR + ILCs.

\section{Clinical and pathologic characteristics}

Detailed demographic, clinical, and histologic data for the three groups are shown in Table 1.

Our series of 38 TN-ILCs comprised exclusively grade II and grade III tumors $(29(76.3 \%)$ and $9(23.7 \%)$, respectively). Patients were all women, with the mean age of 71.7 years (range: $37-92$ years). Twenty of them (52.6\%) had axillary lymph node extension and three $(7.9 \%)$ were metastatic at diagnosis. Twelve patients $(31.6 \%)$ presented with stage I disease, 11 (28.9\%)—-stage II, 12 (31.6\%)stage III, and 3 (7.9\%) with stage IV disease.

We found that TN-ILCs appeared in older patients than HR + ILCs $(P=0.002)$ and TN-IDCs $(P<0.001)$. They were more often single pleomorphic tumors, while HR + ILCs were more multifocal $(P=0.010)$ and more often of the classic subtype $(P=0.003)$. Apocrine differentiation was more prevalent in TN-ILC tumors than in TN-IDCs and
HR + ILCs $(P<0.001)$. Moreover, TN-ILCs had higher nuclear grades than HR + ILCs $(P<0.001)$ but lower mean mitotic counts than TN-IDCs $(P<0.001)$.

Concerning patient management, TN-IDC patients more often underwent lumpectomy than TN-ILC patients among whom mastectomy was more common $(P=0.009)$. No difference in terms of surgical treatment was observed between TN-ILC and HR + ILC patients $(P=0.099)$. As far as the other treatment modalities are concerned, fewer TN-ILC than TN-IDC patients received neoadjuvant chemotherapy $(P=0.009)$ and fewer had radiotherapy compared to TN-IDC $(P=0.005)$ and HR + ILC patients $(P=0.014)$.

\section{Immunohistochemical characteristics}

We found that E-cadherin expression was negative in 34 out of 38 TN-ILC cases $(89.5 \%)$ and in 73 out of 76 HR + ILC cases $(96.1 \%)$, while it was expressed by all TN-IDC tumors. SOX10 and EGFR were more frequently expressed by TN-ILC tumors $(3(7.9 \%)$ and $8(21.1 \%)$ out of 38 , respectively) than in HR + ILC cases (only one of 76 cases expressed EGFR $(1.3 \% ; P<0.001)$, and none showed SOX10 expression $(P=0.035))$, but less frequently than in TN-IDC tumors $(36(47.4 \% ; P<0.001)$ and 52 of 76 (68.4\%; $P<0.001$ ), respectively). CK5/6 was also less frequently expressed in TN-ILC than in TN-IDC tumors (3 $(7.9 \%)$ and 49 (64.5\%) cases, respectively; $P<0.001)$. The mean Ki67 proliferation index was lower in TN-ILC compared to TN-IDC cases $(16.6 \%$ versus $38.9 \%$; $P<0.001)$, while AR positivity was more prevalent in TN-ILC than in versus TN-IDC tumors (36 (94.7\%) versus 38 (50.0\%); $P<$ $0.001)$. Detailed results of all the immunohistochemical analyses are presented in Table 2 and the main findings are illustrated in Fig. 1.

\section{Genomic profiles}

Somatic mutation profiles were available for 26 TN-ILCs, 36 TN-IDCs, and $37 \mathrm{HR}+$ ILCs. We found that TN-ILCs harbored some recurrent pathogenic alterations typical for breast cancers. Besides an expected high rate of $\mathrm{CDHI}$ mutations $(50.0 \%$ ), we found that $61.5 \%$ of TN-ILCs were mutated in at least one of the three key genes of the phosphatidylinositol 3-kinase (PI3K) pathway, mainly PIK3CA (46.2\%), the negative regulator PTEN (11.5\%) and the downstream effector kinase AKT1 (11.5\%). Moreover, several transcription regulators were recurrently altered, such as GATA3 (11.5\%), TBX3 (11.5\%), FOXA1 (7.7\%), and chromatin regulatory factor ARIDIA (7.7\%). ERBB2 and $E R B B 3$, members of the human EGFR family, were mutated in $26.9 \%$ and $3.8 \%$ of cases, respectively. TP53 and BRCAl alterations, typical for TNBC, were found in $15.4 \%$ and $0.0 \%$ of TN-ILCs, respectively (Fig. 2). The 
Table 1 Clinical and pathologic characteristics of triple-negative invasive lobular carcinomas (TN-ILC) compared to two control groups.

\begin{tabular}{|c|c|c|c|}
\hline & \multirow[b]{2}{*}{$\begin{array}{l}\text { TN-ILC } \\
(n=38)\end{array}$} & \multicolumn{2}{|c|}{ Controls } \\
\hline & & $\begin{array}{l}\text { TN-IDC } \\
(n=76)\end{array}$ & $\begin{array}{c}\mathrm{HR}+\mathrm{ILC} \\
(n=76)\end{array}$ \\
\hline \multicolumn{4}{|l|}{ Age at diagnosis (years) } \\
\hline Mean \pm standard deviation & $71.7 \pm 12.7$ & $59.2 \pm 15.5$ & $63.7 \pm 12.6$ \\
\hline Median [min-max] & $74.0[37.0-92.0]$ & $57.0[32.0-100.0]$ & $64.5[37.0-91.0]$ \\
\hline$P$ values for comparisons with TN-ILC & & $<0.001$ & 0.002 \\
\hline \multicolumn{4}{|l|}{ Unifocal tumor } \\
\hline Yes & $36(94.7 \%)$ & $69(90.8 \%)$ & $57(75.0 \%)$ \\
\hline No & $2(5.3 \%)$ & $7(9.2 \%)$ & $19(25 \%)$ \\
\hline$P$ values for comparisons with TN-ILC & & 0.716 & 0.010 \\
\hline \multicolumn{4}{|l|}{ Tumor size $(\mathrm{cm})$} \\
\hline Mean \pm Standard Deviation & $4.1 \pm 3.5$ & $2.9 \pm 1.9$ & $2.9 \pm 2.3$ \\
\hline Median [min-max] & $2.8[0.4-15.0]$ & $2.5[0.5-10.0]$ & $2.0[0.4-10.0]$ \\
\hline$P$ values for comparisons with TN-ILC & & 0.291 & 0.095 \\
\hline \multicolumn{4}{|l|}{ Glandular differentiation } \\
\hline I & $0(0.0 \%)$ & $2(2.6 \%)$ & $0(0.0 \%)$ \\
\hline II & $0(0.0 \%)$ & $30(39.5 \%)$ & $1(1.3 \%)$ \\
\hline III & $38(100 \%)$ & $44(57.9 \%)$ & $75(98.7 \%)$ \\
\hline$P$ values for comparisons with TN-ILC & & $<0.001$ & 1.000 \\
\hline \multicolumn{4}{|l|}{ Nuclear grade } \\
\hline I & $0(0.0 \%)$ & $0(0.0 \%)$ & $12(15.8 \%)$ \\
\hline II & $18(47.4 \%)$ & $26(34.2 \%)$ & $47(61.8 \%)$ \\
\hline III & $20(52.6 \%)$ & $50(65.8 \%)$ & $17(22.4 \%)$ \\
\hline$P$ values for comparisons with TN-ILC & & 0.174 & $<0.001$ \\
\hline \multicolumn{4}{|l|}{ Mitosis score } \\
\hline I & $29(76.3 \%)$ & $24(31.6 \%)$ & $51(67.1 \%)$ \\
\hline II & $5(13.2 \%)$ & $22(28.9 \%)$ & $11(14.5 \%)$ \\
\hline III & $4(10.5 \%)$ & $30(39.5 \%)$ & $14(18.4 \%)$ \\
\hline$P$ values for comparisons with TN-ILC & & $<0.001$ & 0.512 \\
\hline \multicolumn{4}{|l|}{ Mitosis index $\left(/ \mathrm{mm}^{2}\right)$} \\
\hline Mean \pm SD & $2.6 \pm 3.6$ & $8.0 \pm 8.0$ & $2.7 \pm 3.6$ \\
\hline Median [min-max] & $0.9[0.0-14.1]$ & $5.2[0.4-40.6]$ & $0.9[0.4-18.4]$ \\
\hline$P$ values for comparisons with TN-ILC & & $<0.001$ & 0.509 \\
\hline \multicolumn{4}{|l|}{ Histologic subtype } \\
\hline Classical & $19(50.0 \%)$ & - & $59(77.6 \%)$ \\
\hline Pleomorphic & $19(50.0 \%)$ & - & $17(22.4 \%)$ \\
\hline$P$ values for comparisons with TN-ILC & & - & 0.003 \\
\hline \multicolumn{4}{|l|}{ Apocrine differentiation } \\
\hline Yes & $23(60.5 \%)$ & $11(14.5 \%)$ & $20(26.3 \%)$ \\
\hline No & $15(39.5 \%)$ & $65(85.5 \%)$ & $56(73.7 \%)$ \\
\hline$P$ values for comparisons with TN-ILC & & $<0.001$ & $<0.001$ \\
\hline \multicolumn{4}{|l|}{ Lymphovascular emboli } \\
\hline Yes & $15(39.5 \%)$ & $24(31.6 \%)$ & $20(26.3 \%)$ \\
\hline No & $23(60.5 \%)$ & $52(68.4 \%)$ & $56(73.7 \%)$ \\
\hline$P$ values for comparisons with TN-ILC & & 0.402 & 0.151 \\
\hline \multicolumn{4}{|l|}{ Necrosis } \\
\hline Yes & $6(15.8 \%)$ & $22(28.9 \%)$ & $3(3.9 \%)$ \\
\hline
\end{tabular}


Table 1 (continued)

\begin{tabular}{|c|c|c|c|}
\hline & \multirow[b]{2}{*}{$\begin{array}{l}\text { TN-ILC } \\
(n=38)\end{array}$} & \multicolumn{2}{|c|}{ Controls } \\
\hline & & $\begin{array}{l}\text { TN-IDC } \\
(n=76)\end{array}$ & $\begin{array}{c}\mathrm{HR}+\mathrm{ILC} \\
(n=76)\end{array}$ \\
\hline No & $32(84.2 \%)$ & $54(71.1 \%)$ & $73(96.1 \%)$ \\
\hline$P$ values for comparisons with TN-ILC & & 0.124 & 0.058 \\
\hline \multicolumn{4}{|l|}{ Surgical treatment } \\
\hline Lumpectomy & $9(23.7 \%)$ & $41(53.9 \%)$ & $33(43.4 \%)$ \\
\hline Mastectomy & $25(65.8 \%)$ & $30(39.5 \%)$ & $39(51.3 \%)$ \\
\hline None & $4(10.5 \%)$ & $5(6.6 \%)$ & $4(5.3 \%)$ \\
\hline$P$ values for comparisons with TN-ILC & & 0.009 & 0.099 \\
\hline \multicolumn{4}{|l|}{ Chemotherapy } \\
\hline Neoadjuvant & $3(7.9 \%)$ & $25(32.9 \%)$ & $4(5.3 \%)$ \\
\hline Adjuvant & $14(36.8 \%)$ & $29(38.2 \%)$ & $29(38.2 \%)$ \\
\hline Exclusive & $4(10.5 \%)$ & $6(7.9 \%)$ & $1(1.3 \%)$ \\
\hline None & $17(44.7 \%)$ & $16(21.1 \%)$ & $42(55.3 \%)$ \\
\hline$P$ values for comparisons with TN-ILC & & 0.009 & 0.125 \\
\hline \multicolumn{4}{|l|}{ Breast radiotherapy } \\
\hline Yes & $21(55.3 \%)$ & $61(80.3 \%)$ & $59(77.6 \%)$ \\
\hline No & $17(44.7 \%)$ & $15(19.7 \%)$ & $17(22.4 \%)$ \\
\hline$P$ values for comparisons with TN-ILC & & 0.005 & 0.014 \\
\hline \multicolumn{4}{|l|}{ Lymph node radiation } \\
\hline Yes & $14(36.8 \%)$ & $33(43.4 \%)$ & $34(44.7 \%)$ \\
\hline No & $24(63.2 \%)$ & $43(56.6 \%)$ & $42(55.3 \%)$ \\
\hline$P$ values for comparisons with TN-ILC & & 0.501 & 0.421 \\
\hline
\end{tabular}

$H R+I L C$ hormone receptor-positive invasive lobular carcinoma, $T N-I D C$ triple-negative invasive ductal carcinoma.

Statistically significant $p$-values are in italic.

Statistically significant $p<0.05$ values are in bold-italic.

prevalence of mutations in genes known to be involved in breast cancer compared between these three tumor subtypes is shown in Supplementary Table S2, and a complete list of the pathogenic and likely pathogenic variants found in these tumors is provided in the Supplementary Table S3.

We found 177 genes that were specifically (significantly) altered in TN-ILCs (Supplementary Table S4). As expected, TN-ILCs were enriched for $\mathrm{CDH} 1$ mutations compared to TN-IDCs $(50.0 \%$ versus $11.1 \% ; P<0.001)$, while TP53 alterations were less prevalent $(15.4 \%$ versus $66.7 \% ; P<$ $0.001)$. Moreover, we observed a trend for a higher prevalence of the PI3K network alterations and of ERBB2 mutations in TN-ILCs, neither of the two, however, has reached statistical significance.

Among other altered genes, we focused on the Estrogen-Related Receptor Alpha (ESRRA) gene encoding an orphan nuclear receptor that shares structural homology with the estrogen receptor $\alpha / \beta(E R \alpha / \beta)[38,39]$. ESRRA was altered in $53.8 \%$ of TN-ILCs, with three specific recurrent hotspot mutations in the nuclear receptor ligandbinding domain: p.R352P (found in $46.2 \%$ of TN-ILCs),
p.R376L (50.0\%), and p.L385P (50.0\%). Of note, none of these three hotspots was found to be mutated in controlsESRRA alterations in controls were fewer $(P<0.001)$ and non-recurrent. All ESRRA gene variants identified in TNILCs compared to control groups are illustrated in Fig. 3 and detailed in Supplementary Table S5. Interestingly, ESRRA-mutant cases were more frequently pleomorphic $(P=0.006)$ with higher nuclear grades $(P=0.006)$, and tended to have higher proliferation indexes $(P=0.072)$ than ESRRA-wildtype tumors. Detailed clinical, pathologic, and immunohistochemical features of ESRRA-mutant and -wildtype TN-ILCs are presented in Supplementary Table 6.

\section{Transcriptomic profiles}

The pathway enrichment analysis highlighted differential activation of several signaling pathways. Compared to HR + ILCs, TN-ILCs showed overexpression of genes under the control of the AR transcription factor $(P<0.001$; Fig. 4A) as well as the enrichment of the Regulation of Ras 
Table 2 Immunohistochemical characteristics of triple-negative invasive lobular carcinomas (TN-ILC) compared to two control groups.

\begin{tabular}{|c|c|c|c|}
\hline \multirow{2}{*}{ Markers } & \multirow[b]{2}{*}{$\begin{array}{l}\text { TN-ILC } \\
(n=38)\end{array}$} & \multicolumn{2}{|c|}{ Controls } \\
\hline & & $\begin{array}{l}\text { TN-IDC } \\
(n=76)\end{array}$ & $\begin{array}{c}\mathrm{HR}+\mathrm{ILC} \\
(n=76)\end{array}$ \\
\hline \multicolumn{4}{|l|}{ E-cadherin } \\
\hline Positive & $4(10.5 \%)$ & $76(100 \%)$ & $3(3.9 \%)$ \\
\hline Negative & $34(89.5 \%)$ & $0(0.0 \%)$ & $73(96.1 \%)$ \\
\hline $\begin{array}{l}P \text { values for } \\
\text { comparisons with } \\
\text { TN-ILC }\end{array}$ & & $<0.001$ & 0.219 \\
\hline \multicolumn{4}{|l|}{ AR } \\
\hline Positive & $36(94.7 \%)$ & $38(50.0 \%)$ & $75(98.7 \%)$ \\
\hline Negative & $2(5.3 \%)$ & $38(50.0 \%)$ & $1(1.3 \%)$ \\
\hline $\begin{array}{l}P \text { values for } \\
\text { comparisons with } \\
\text { TN-ILC }\end{array}$ & & $<0.001$ & 0.257 \\
\hline \multicolumn{4}{|l|}{ CK5/6 } \\
\hline Positive & $3(7.9 \%)$ & $49(64.5 \%)$ & $3(3.9 \%)$ \\
\hline Negative & $35(92.1 \%)$ & $27(35.5 \%)$ & $73(96.1 \%)$ \\
\hline $\begin{array}{l}P \text { values for } \\
\text { comparisons with } \\
\text { TN-ILC }\end{array}$ & & $<0.001$ & 0.399 \\
\hline \multicolumn{4}{|l|}{ EGFR } \\
\hline Positive & $8(21.1 \%)$ & $52(68.4 \%)$ & $1(1.3 \%)$ \\
\hline Negative & $30(78.9 \%)$ & $24(31.6 \%)$ & $75(98.7 \%)$ \\
\hline $\begin{array}{l}P \text { values for } \\
\text { comparisons with } \\
\text { TN-ILC }\end{array}$ & & $<0.001$ & $<0.001$ \\
\hline \multicolumn{4}{|l|}{ SOX10 } \\
\hline Positive & $3(7.9 \%)$ & $36(47.4 \%)$ & $0(0.0 \%)$ \\
\hline Negative & $35(92.1 \%)$ & $40(52.6 \%)$ & $76(100 \%)$ \\
\hline $\begin{array}{l}P \text { values for } \\
\text { comparisons with } \\
\text { TN-ILC }\end{array}$ & & $<0.001$ & 0.035 \\
\hline \multicolumn{4}{|l|}{ Ki67 (\%) } \\
\hline $\begin{array}{l}\text { Mean } \pm \text { Standard } \\
\text { Deviation }\end{array}$ & $16.6 \pm 19.6$ & $38.9 \pm 24.9$ & $13.7 \pm 10.7$ \\
\hline Median $[\min -\max ]$ & $\begin{array}{c}10.0 \\
{[1.0-90.0]}\end{array}$ & $\begin{array}{c}32.5 \\
{[2.0-90.0]}\end{array}$ & $\begin{array}{c}10.0 \\
{[1.0-50.0]}\end{array}$ \\
\hline $\begin{array}{l}P \text { values for } \\
\text { comparisons with } \\
\text { TN-ILC }\end{array}$ & & $<0.001$ & 0.918 \\
\hline
\end{tabular}

$A R$ androgen receptor, $C K 5 / 6$ cytokeratin 5/6, EGFR epidermal growth factor receptor, $H R+I L C$ hormone receptor-positive invasive lobular carcinoma; $T N-I D C$ triple-negative invasive ductal carcinoma. Statistically significant $p$-values are in italic.

Statistically significant $p<0.05$ values are in bold-italic.

familly activation $(P=0.017)$ and of the Syndecan-1mediated signaling pathways $(P=0.036)$. In contrast, IL2 signaling pathways mediated by STAT5 and PI3K were downregulated $(P=0.007$ and $P=0.009$, respectively). Moreover, TN-ILCs presented MAPK1 and ERBB3 activation $(P=0.013$ and $P=0.003$, respectively; Fig. $4 \mathrm{~B})$, a higher expression of AKT1-related genes $(P=0.008$; Fig. 4B) and a lower expression of genes regulated by ESR 1 $(P<0.001$; Fig. 4A). Otherwise, compared to TN-IDCs, TN-ILCs showed enriched AR activity co-regulation $(P=0.038)$ and FOXA1 network $(P=0.002)$ associated with overexpression of genes under the control of ESR 1 and PPARG transcription factors $(P=0.009$ and $P=0.023$, respectively; Fig. 4A), whereas the Stabilization and expansion of the E-cadherin and the Syndecan-1-mediated signaling pathways were downregulated $(P=0.006$ and $P=0.007$, respectively). We also found AKT1, RAF1 (c-Raf), and CDK8 activation $(P=0.005)$, and a lower expression of ERBB3 $(P=0.010)$, AKT2 $(P=0.010)$, and BRAF $(P=0.002$; Fig. 4B). For a list of all genes whose expression was found to be significantly altered in TN-ILCs in comparison to HR + ILCs and/or TN-IDCs, see Supplementary Tables S7 and S8, respectively.

\section{Molecular subtype classifications}

Intrinsic subtype classification following RNA sequencing resulted in 12 out of $28(42.9 \%)$ TN-ILC tumors being classified as luminal A, $11(39.3 \%)$ as HER2-enriched, 2 (7.1\%) as Normal breast-like, and only $3(10.7 \%)$ as basal. In comparison, $22(62.9 \%)$ of 35 TN-IDC-tumors were classified as basal and $32(91.5 \%)$ of $35 \mathrm{HR}+$ ILC tumors were considered as Luminal, either A $(n=15)$ or $\mathrm{B}(n=17$; $P<0.001)$. When using the Lehmann's TNBC subtype classification, most of the TN-ILCs fell within the Luminal androgen receptor (LAR; $n=11$ ) or the ER-positive (ESR1-activated) category $(n=9)$. Overall, $71.4 \% \mathrm{TN}$ ILCs were in either of these two categories, while among TN-IDC it was only $8.6 \%$ of cases. Consistently, a significant proportion of TN-IDC tumors (40\%) were classified as basal-like (10 as Basal-like 1, and 4 as Basal-like 2), whereas it was the case of only $7.2 \%$ among TN-ILCs $(P<0.001)$. The main results for molecular subtype classifications are summarized in Table 3 and detailed case-bycase classifications are listed in Supplementary Table S9. Of note, no statistically significant association was observed between molecular subtypes and the ESRRA mutational status in our series. However, this may be due to small numbers of tumors by subtype.

\section{Progression and survival}

The median follow-up of patients was 63.5 months (range: 0-226 months). Three TN-ILC patients (7.9\%) had local recurrence during the follow-up, eight $(22.9 \%)$ developed 

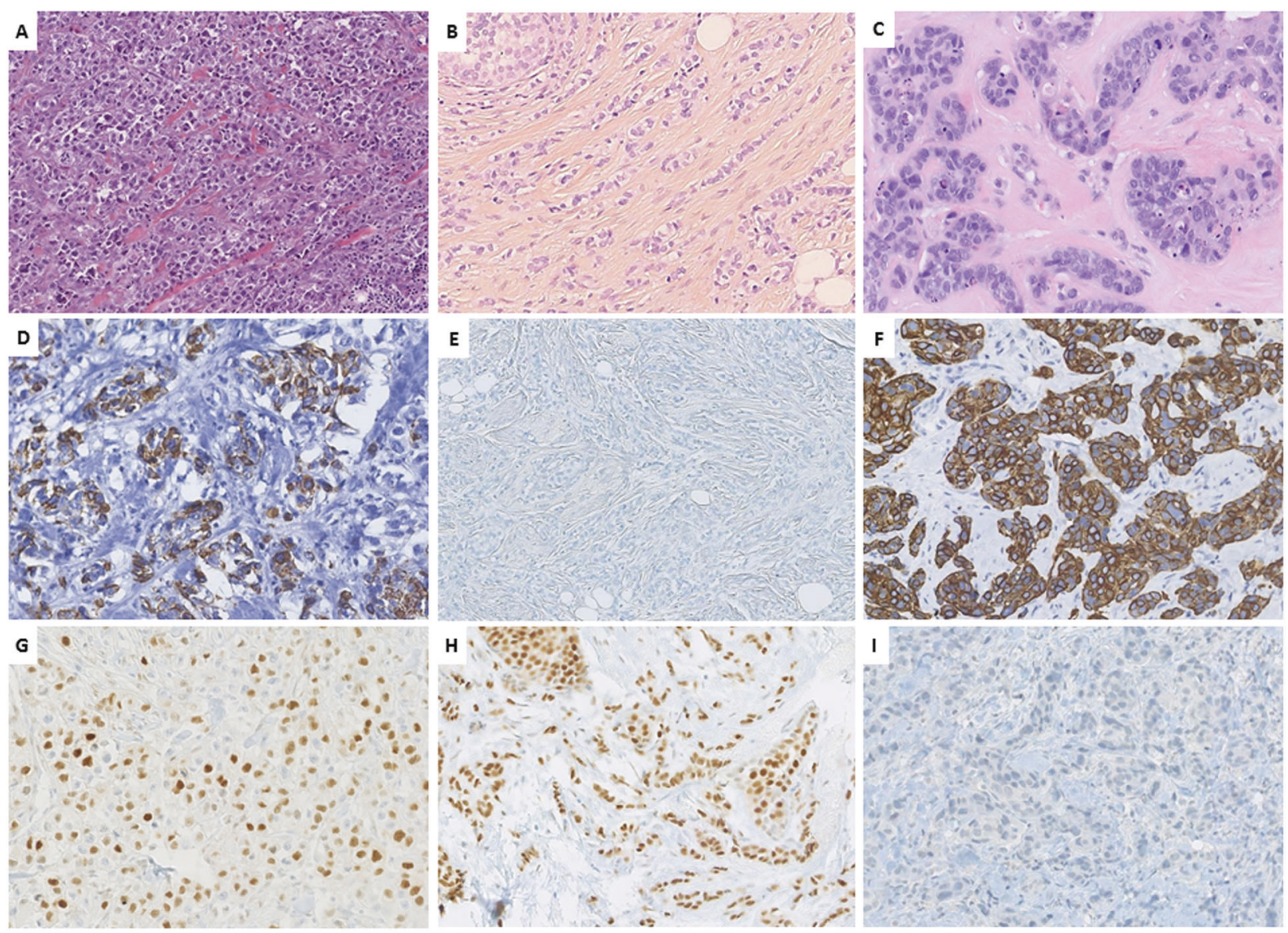

Fig. 1 Morphological and main immunohistochemical features of triple-negative invasive breast lobular carcinoma (TN-ILC) compared to control tumors. Mild power view of pleomorphic TN-ILC (A) compared to classic hormone receptor-positive invasive lobular carcinoma (HR + ILC) (B) and triple-negative invasive ductal

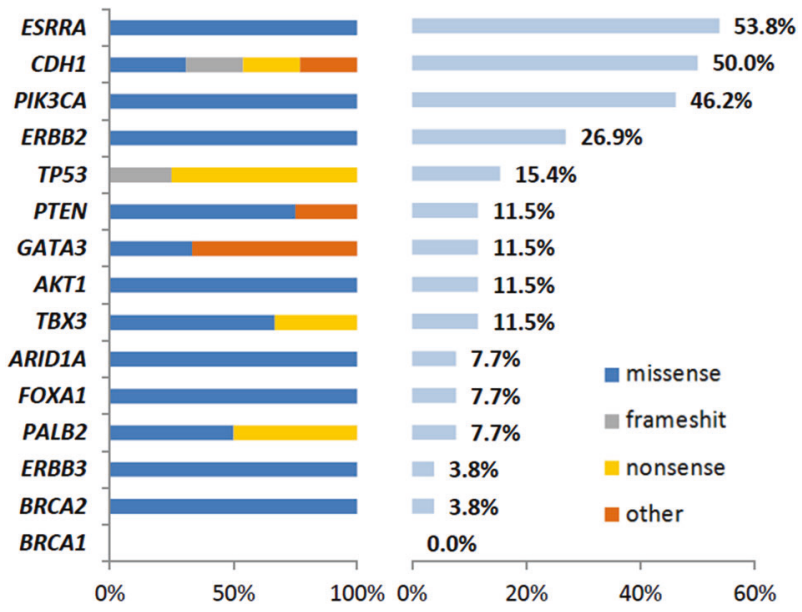

Fig. 2 The genomic landscape of triple-negative invasive lobular carcinoma of the breast. DNA from 26 primary tumors was analyzed by whole-exome sequencing. The prevalence and type of recurrent alterations are shown. carcinoma (TN-IDC) (C) (H\&E-saffron) with partial membranous immunostaining for cytokeratin $5 / 6$ in TN-ILC (D) compared to negativity in HR + ILC (E) and diffuse and strong expression in TNIDC $(\mathbf{F})$. Diffuse nuclear positivity for androgen receptor in TN-ILC (G) and HR + ILC (H) compared to negativity in TN-IDC (I).

metastases, and $10(26.3 \%)$ died of the disease. Median times to local recurrence and distant metastasis were 56 (22-66) and 5 (1-59) months, respectively, compared to 12.5 (9-92) and 15 months (5-40) for TN-IDC, and 71 (71-71) and 33.5 (23-114) months for HR + ILC patients.

Five-year distant metastasis-free survival for initially non-metastatic patients and 5-year overall survival for all patients were significantly worse for TN-ILC patients than for those with HR + ILC: 68.7\% (95\% CI 48.86-82.14) and 67.9\% (95\% CI: 49.10-81.02) for TN-ILC patients compared to $85.3 \%$ (95\% CI: 74.30-91.83; $P=0.039$ ) and 84.3\% (95\%CI: 73.32-91.02; $P=0.047$ ) for those with HR + ILC (Fig. 5). The same association was observed for progression-free and locoregional progression-free survivals ( $P=0.015$ and $P=0.020$, respectively). However, the survival did not differ between TN-ILC and TN-IDC patients (Fig. 5). Moreover, there were no differences in survival between ESRRA-mutant and ESRRA-wildtype TNILC cases (data not shown). 


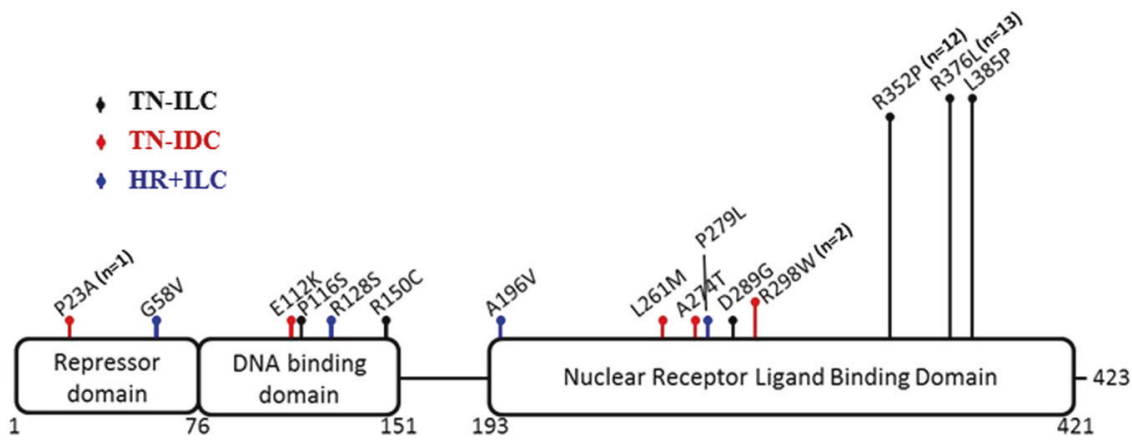

Fig. 3 Distribution of ESRRA genetic alterations in triple-negative invasive lobular carcinoma compared to two control groups. The location of alterations identified by whole-exome sequencing in different regions of the gene is shown. The bar heights are proportionate to the number of mutant cases $(n)$. TN-ILC triple-negative invasive lobular carcinoma, TN-IDC triple-negative invasive ductal carcinoma, HR + ILC hormone receptor-positive invasive lobular carcinoma.
Univariate and multivariate analyses determined two parameters independently associated with survival in $\mathrm{TN}$ ILC patients: $\mathrm{T}$ stage $(\mathrm{HR}=7.95$; 95\% CI: 2.29-27.57; $P=0.001)$ and $\mathrm{M}$ stage $(\mathrm{HR}=3.57$; $95 \% \mathrm{CI}: 1.01-12.68$; $P=0.049)$.

\section{Discussion}

TNBCs are a small subset of breast cancers which are not sensitive to endocrine or anti-HER2 therapy and are usually associated with poor prognosis $[9,10]$. They encompass distinct tumor subtypes with different biological characteristics and prognosis, and different, often limited, responsiveness to chemotherapy $[11,18,40]$. With the advent of new technologies, much hope is pinned to the potential of targeted therapies, with new molecular targets emerging from recent studies [41, 42]. However, most of the previous studies focused on IDC-NST, while TN-ILC remains poorly known. In this study, we characterized 38 primary TN-ILCs (accounting for $0.1 \%$ of all breast cancers diagnosed in our institutions within the study period) in comparison with phenotypically close tumors: TN-IDCs and HR + ILCs, matched by stage and grade. In accordance with the results of others, we found that TN-ILCs were more prevalent in elderly patients than TN-IDCs and HR + ILCs [19], and, appeared more unifocal and more often pleomorphic on microscopic examination, with higher nuclear grades than HR + ILCs [17, 19].

TNBCs often overlap with basal-like breast carcinomas (BLBCs) as defined by gene expression profiling. However, $18-40 \%$ of BLBCs do express either hormone receptors or HER2, and therefore cannot be classified as TNBCs $[43,44]$. BLBCs are usually defined as tumors negative for both hormone receptors and HER2, and at the same time positive for at least one basal marker as defined by immunohistochemistry [45]. In our study, we used a panel of antibodies known as basal markers, including both established (CK5/6 and EGFR) and recently suggested markers (SOX10) $[45,46]$. We found that only a small subset of TNILCs $(26.3 \%$ versus $93.4 \%$ of TN-IDCs) could be classified as BLBCs, which is consistent with previous reports [19]. Our molecular analyses confirmed these results, as most of TN-ILCs were classified as luminal A or HER2-enriched, and not as basal tumors, according to the intrinsic molecular classification.

TNBCs are a heterogeneous group of tumors that exhibit substantial differences in terms of morphological features, biological behavior, and gene expression profiles. Several studies have focused on the molecular subtyping of TNBCs and the identification of putative therapeutic targets. Based on gene expression profiles, Lehmann et al. [11], and others [12-14] recently identified different TNBC subtypes, all including a luminal androgen receptor (LAR) subtype, mainly characterized by increased AR signaling and associated with elderly age, decreased relapse-free survival and lower chemotherapy responsiveness $[11-14,27]$. AR, a member of the nuclear receptor family and a liganddependent transcription factor, is frequently expressed in HR-positive breast cancers (70-90\%), while the reported prevalence of its expression in TNBC varies depending on the series (8-53\%) [47]. In our study, ILCs more frequently expressed AR than TN-IDCs (as determined by immunohistochemistry), whatever the hormonal status, which is contradictory to previous data [17].

Interestingly, since most LAR tumors are classified as luminal or HER2-enriched by intrinsic molecular classification, and none as basal [11, 27], most of our TN-ILC cases which express AR, but not basal markers, could belong to the LAR subtype. This hypothesis is further supported by our transcriptomic results, showing the enrichment of AR signaling and FOXA1 network pathways in TN-ILCs. FOXA1 may direct AR to sites normally occupied by estrogen receptors in luminal tumors, inducing 

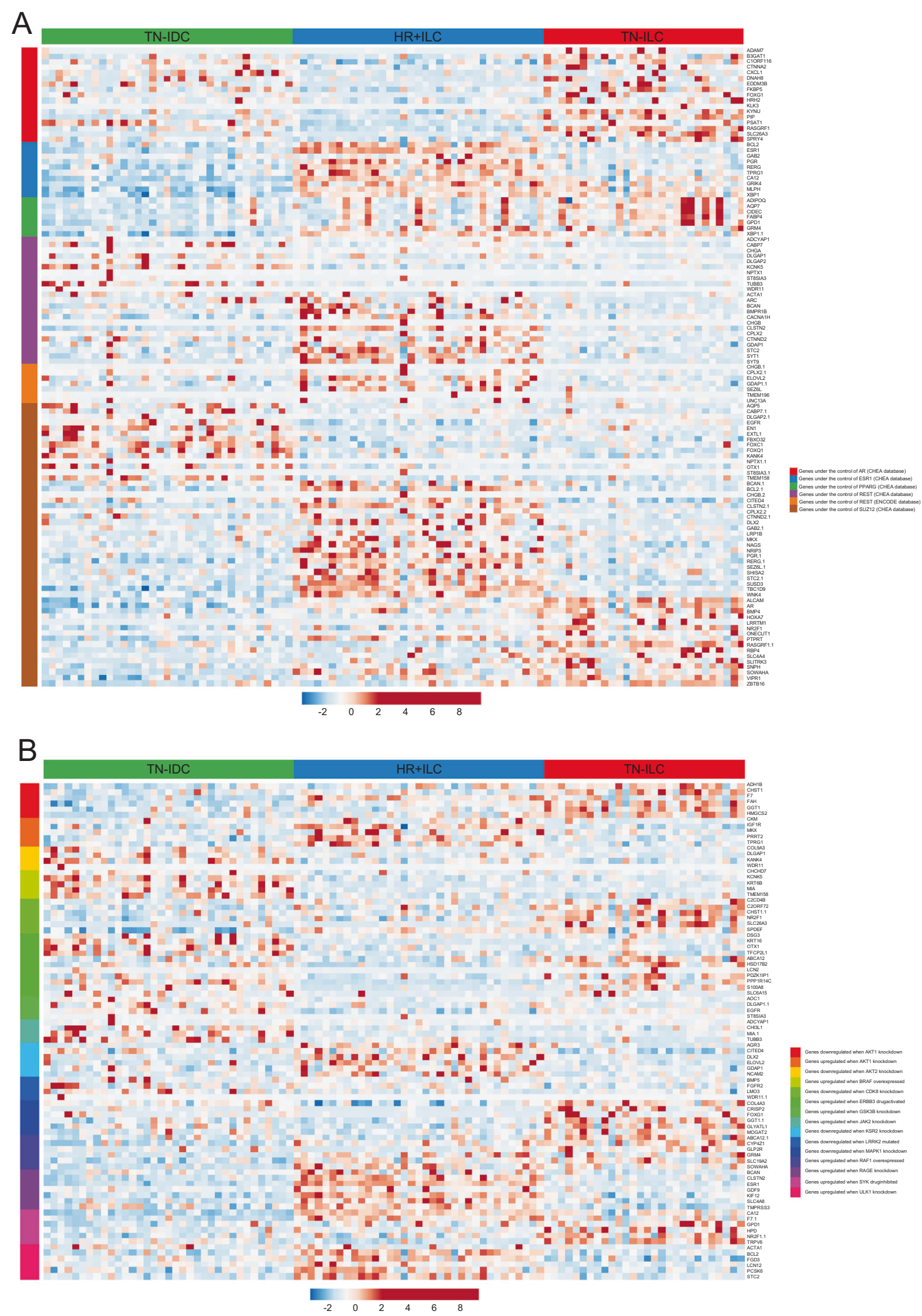

an estrogen-like gene program that stimulates proliferation, and its expression was previously reported in the LAR subtype [11, 13, 48]. Interestingly, although TN-ILCs were confirmed to be TN, they present an overexpression of ESR1-regulated genes, which is typical for hormonepositive breast tumors, compared to TN-IDCs. These 
Fig. 4 The transcriptomic profile of triple-negative invasive breast lobular carcinoma (TN-ILC) compared to control tumors. Gene expression was analyzed in 28 primary TN-ILCs by RNA sequencing and compared to control tumors. The heatmaps show the expression of genes found to be significantly up- or downregulated in TN-ILC compared to controls, by the transcription factor involved in their regulation $(\mathbf{A})$, and by related kinase perturbations $(\mathbf{B})$. Gene expression levels were assessed by calculating $Z$-scores. TN-IDC triple-negative invasive ductal carcinoma, HR + ILC hormone receptor-positive invasive lobular carcinoma.

Table 3 Intrinsic and Lehmann's TNBC molecular subtype classifications based on RNA sequencing for triple-negative invasive lobular carcinomas (TN-ILC) compared to two control groups.

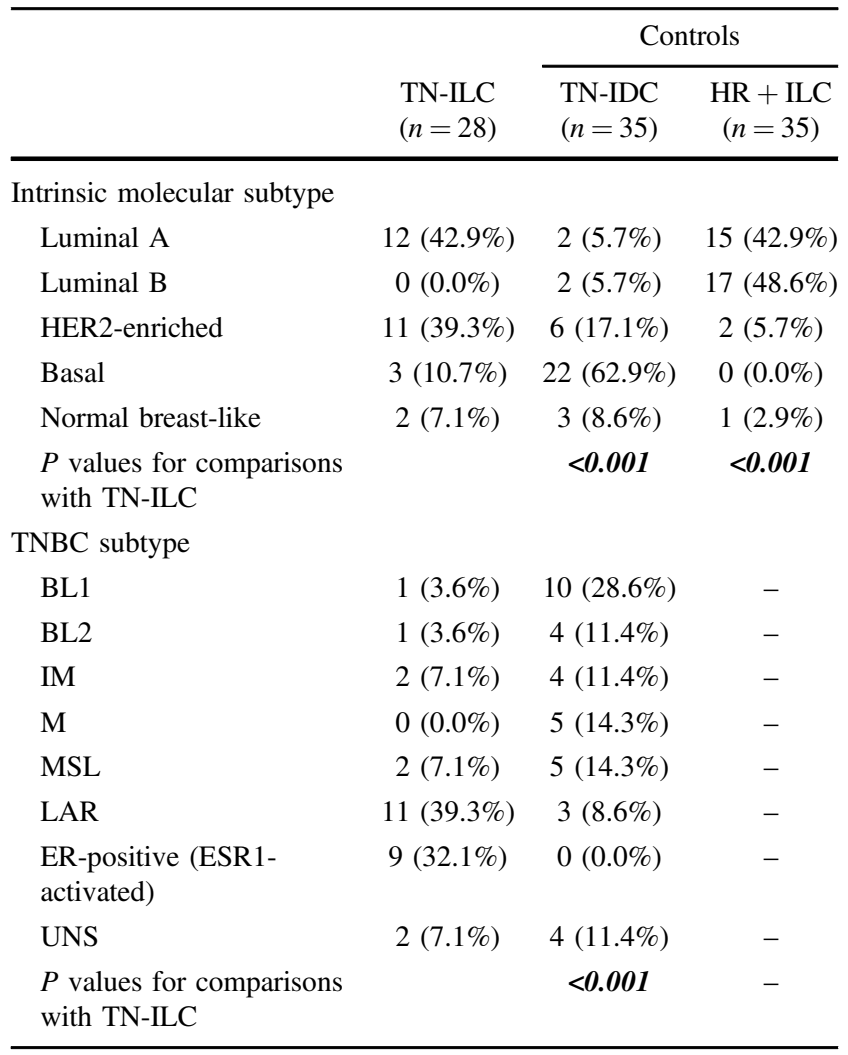

$B L 1$ basal-like 1, $B L 2$ basal-like 2; $E R$ estrogen receptor-positive, $H R$ $+I L C$ hormone receptor-positive invasive lobular carcinoma, IM immunomodulatory, $L A R$ luminal androgen receptor, $M$ mesenchymal, $M S L$ mesenchymal stem-like, $T N B C$ triple-negative breast carcinoma, $T N-I D C$ triple-negative invasive ductal carcinoma, UNS unstable.

Statistically significant $\mathrm{p}<0.05$ values are in bold-italic.

results are also consistent with previous studies showing enrichment in several hormonally regulated pathways, including estrogen signaling pathway, in LAR tumors compared to others TNBC subtypes [11, 13, 14]. The peroxisome proliferator-activated receptor (PPAR) signaling pathway is also reported to be significantly increased in the LAR subtype. Our subtyping results obtained with the TNBC-type online tool provided by Lehmann further confirm this hypothesis, as most of our TN-ILCs were classified as LAR or ESR1-activated tumors.
Consequently, this constitutes molecular evidence for the activation of the AR and ER pathways (which may induce sensitivity to traditional antiestrogen therapies and antiandrogens) in tumors defined as HR-negative by immunohistochemistry. Of note, despite the fact that AR expression in TN-ILCs and HR + ILCs appeared to be identical by immunohistochemistry, our RNA-sequencing results showed overexpression of the AR pathway in TN-ILCs compared to HR + ILCs, but with lower expression of ESR1-regulated genes.

Comparative exome analyses highlighted a peculiar molecular landscape in TN-ILC. In addition to the expected increased prevalence of $\mathrm{CDH} 1$ alterations, TN-ILCs seemed to have a higher prevalence of PI3K pathway gene alterations and $E R B B 2$ mutations than the other two tumor types. These findings are consistent with those of previous studies which showed a higher PIK3CA mutation rate in ARpositive TNBCs [11] and higher rates of ERBB2 mutations in the LAR subtype tumors [12]. In contrast, TP53 and BRCA1 mutations, characteristics for TNBC, were not found to be enriched in TN-ILC. Interestingly, we found specific ESRRA gene alterations in these tumors: TN-ILCs harbored three recurrent ESRRA hotspot mutations: R352P, $\mathrm{R} 376 \mathrm{~L}$, and L385P, none of which was present in controls. These new mutations, never described in breast cancer to date, appear to be a distinctive feature of TN-ILC.

ESRRA encodes Estrogen-Related Receptor alpha (ERR $\alpha$ ). ERR $\alpha$, co-regulated by PPAR $\gamma$ co-activator- $1 \alpha$ and $\beta$, is considered to be a master regulator of cellular energy metabolism [49]. Although ERR $\alpha$ shares a high degree of homology with $E R \alpha / \beta$, it does not bind estrogens or antiestrogens, and its activation mechanism and its functions are different [38]. In breast cancer, ERR $\alpha$ seems to partly enhance tumor development accommodating energy demands of TCs. It may also activate the ER $\alpha$ pathway by inducing the transcription of some genes normally controlled by estrogens $[38,39,49,50]$. Interestingly, cross-talks with the AR signaling have been demonstrated in prostate cancer [51]. In TNBC, high ERR $\alpha$ expression has been shown to be associated with poor prognosis and at the same time predict response to tamoxifen [52]. Our results are consistent with these findings: we found that $\mathrm{TN}$ ILCs with at least one ESRRA hotspot mutation had a more aggressive phenotype, with more nuclear atypia and pleomorphism than tumors without these alterations. Taking all this into account, we explored the ESRRA protein expression in an attempt to correlate genomic mutations with protein expression determined by immunohistochemistry, but without convincing results in this small cohort (data not shown). However, the possibility that TN-ILCs carrying these ESRRA mutations are sensitive to endocrine therapy is definitely worth more attention and should be verified using larger cohorts. 

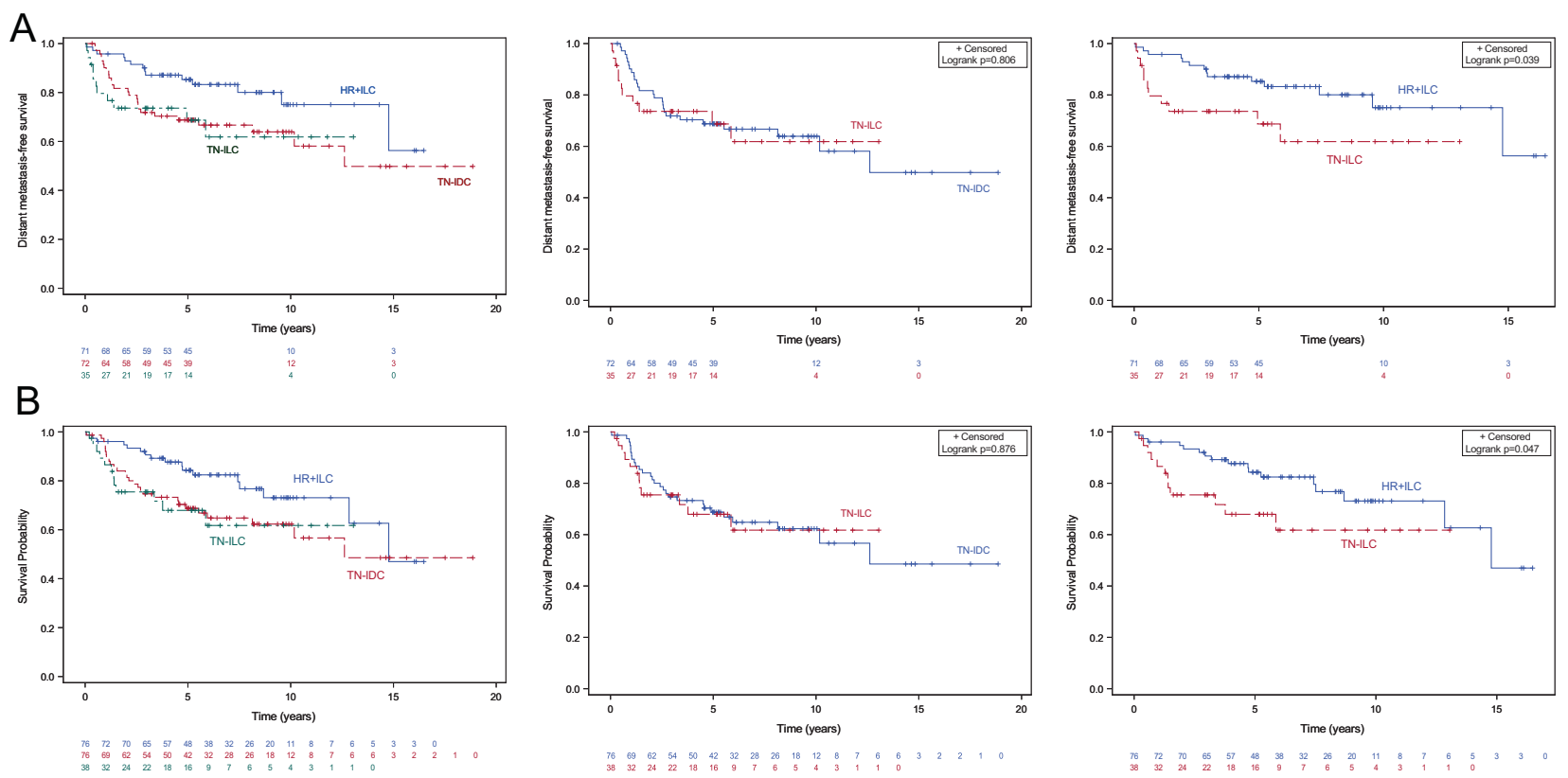

Fig. 5 Clinical outcome of triple-negative invasive breast lobular carcinoma (TN-ILC) compared to control tumors. Kaplan-Meier curves of distant metastasis-free (A) and overall survival (B) for TN-ILC compared to controls. The tables below the curves indicate the numbers of patients at risk. HR + ILC: hormone receptor-positive invasive lobular carcinoma; TN-IDC: triple-negative invasive ductal carcinoma.
In our series, TN-ILC patients more frequently had mastectomy than lumpectomy compared to TN-IDC patients, possibly due to difficulties in defining tumor margins [5]. They also less often received chemotherapy. This may have been due to older age and age-associated comorbidities making the patients unsuitable for administration of this treatment modality. It also goes in line with the fact that in our series, TN-ILCs were associated with poor outcomes compared to HR + ILCs. This is partly consistent with the results by Iorfida et al. who showed that TN-ILC patients had a worse disease-free survival than luminal A ILC patients [20]. Of note, in our study, the control tumors were matched to TN-ILCs by stage and grade, the two established prognostic factors, so the observed differences in the outcomes can be attributed to different biological characteristics of the tumors from the three groups. Even though the literature data on TN-ILC prognosis differ [17-20], it seems that the absence of targeted therapy for TN-ILC patients, who-unlike those with HR + ILC - cannot benefit from endocrine therapy, may largely contribute to poor prognosis.

Recent studies demonstrated an antitumor effect of AR antagonists in AR + TNBC, with encouraging phase II and III clinical trials results $[11,53,54]$. Since PIK3CA mutations are frequent in $\mathrm{AR}+\mathrm{TNBC}$, combining PI3K inhibitors and $\mathrm{AR}$ antagonists seems to increase treatment effectiveness in patients with these tumors [11, 53]. Synchronous targeting of AR and $\mathrm{PI} 3 \mathrm{~K} / \mathrm{AKT} / \mathrm{mTOR}$ pathway may thus offer an interesting alternative for treating TN-ILC patients. Of note, although immunohistochemical analysis indicates that TN-ILCs are HR-negative breast cancers, estrogen signaling pathway activation associated with highlighting of specific ESRRA mutations in these particular tumors may suggest sensitivity to traditional antiestrogen therapy, and need further consideration [11, 52].

In this retrospective study, we have analyzed clinical, morphologic, immunohistochemical, and molecular characteristics of 38 primary TN-ILC tumors compared to phenotypically similar tumors. TN-ILCs are rare tumors. Even though small sample size is an obvious limitation of our study, it is still-to the best of our knowledge-the largest study of TN-ILC ever reported in the literature. We found that TN-ILCs are unique breast cancers which affect elderly women and have particular characteristics, often in between those of conventional ILC and those of TN-IDC. While morphology, loss of E-cadherin associated with $\mathrm{CDH} 1$ mutations and low proliferation index make them similar to HR + ILC, their aggressive behavior associated with high nuclear grade, pleomorphic subtype and poor prognosis resemble TN-IDC. Moreover, we found that TNILCs presents a transcriptomic profile typical for the LAR TNBC subtype, closely related to AR signaling and associated with frequent alterations in the PI3K network and ERBB2. Finally, we identified new specific ESRRA mutations, never reported in breast cancer to date, which were recurrent in TN-ILCs. Even though these findings should 
still be confirmed using larger cohorts, they may contribute to understanding the biology and pave the way for new therapeutic strategies to treat these particular tumors.

\section{Data availability}

All data analyzed in this study are included in this article or in the associated additional files.

Acknowledgements The authors would like to thank Katarzyna Szymanska (Science to the Point), Ph.D., a medical writer and a certified English translator, for language revision and editorial advice.

Author contributions AnB and LA designed the study. GMG, PA, FB, $\mathrm{CCB}, \mathrm{EDC}, \mathrm{SL}, \mathrm{ID}, \mathrm{HB}, \mathrm{CL}$, and SA provided the patient data. AnB, $\mathrm{RB}$, and LA performed the experiments, analyzed the data and wrote the manuscript. $\mathrm{RB}$ and $\mathrm{CR}$ were responsible for molecular experiments and analysis. AuB was responsible for statistical analysis and final interpretation. AnB and LA supervised the research. All authors critically revised the final version of the manuscript.

Funding The authors do not declare any grant specific for this research from any funding agency from the public, private, or not-for-profit sector.

\section{Compliance with ethical standards}

Conflict of interest The authors declare no competing interests.

Ethical approval The study was approved by the CGFL Research Ethics Committee. CGFL has been authorized to conduct scientific research by relevant French authorities (authorization number $A C$ 2019-3531).

Publisher's note Springer Nature remains neutral with regard to jurisdictional claims in published maps and institutional affiliations.

\section{References}

1. Lakhani, SR, Ellis IO, Schnitt, SJ, Tan, PH, van de Vijver, MJ. WHO classification of tumours of the breast, 5th ed. Lyon: International Agency for Research on Cancer; 2019.

2. Vargas A-C, Lakhani SR, Simpson PT. Pleomorphic lobular carcinoma of the breast: molecular pathology and clinical impact. Future Oncol. 2009;5:233-43.

3. Reed AEM, Kutasovic JR, Lakhani SR, Simpson PT. Invasive lobular carcinoma of the breast: morphology, biomarkers and'omics. Breast Cancer Res. 2015;17:12.

4. Ciriello G, Gatza ML, Beck AH, Wilkerson MD, Rhie SK, Pastore A, et al. Comprehensive molecular portraits of invasive lobular breast cancer. Cell. 2015;163:506-19.

5. Yeatman TJ, Cantor AB, Smith TJ, Smith SK, Reintgen DS, Miller MS, et al. Tumor biology of infiltrating lobular carcinoma. Implic Manag Ann Surg. 1995;222:549-59. discussion 559-561

6. Arpino G, Bardou VJ, Clark GM, Elledge RM. Infiltrating lobular carcinoma of the breast: tumor characteristics and clinical outcome. Breast Cancer Res. 2004;6:R149-R156.

7. Rakha EA, El-Sayed ME, Powe DG, Green AR, Habashy H, Grainge MJ, et al. Invasive lobular carcinoma of the breast: response to hormonal therapy and outcomes. Eur J Cancer. 2008;44:73-83.
8. Pestalozzi BC, Zahrieh D, Mallon E, Gusterson BA, Price KN, Gelber RD, et al. Distinct clinical and prognostic features of infiltrating lobular carcinoma of the breast: combined results of 15 International Breast Cancer Study Group clinical trials. J Clin Oncol. 2008;26:3006-14.

9. Thike AA, Cheok PY, Jara-Lazaro AR, Tan B, Tan P, Tan PH. Triple-negative breast cancer: clinicopathological characteristics and relationship with basal-like breast cancer. Mod Pathol. 2010;23:123-33.

10. Dent R, Trudeau M, Pritchard KI, Hanna WM, Kahn HK, Sawka $\mathrm{CA}$, et al. Triple-negative breast cancer: clinical features and patterns of recurrence. Clin Cancer Res. 2007;13:4429-34.

11. Lehmann BD, Bauer JA, Chen X, Sanders ME, Chakravarthy AB, Shyr Y, et al. Identification of human triple-negative breast cancer subtypes and preclinical models for selection of targeted therapies. J Clin Investig. 2011;121:2750-67.

12. Jiang Y-Z, Ma D, Suo C, Shi J, Xue M, Hu X, et al. Genomic and transcriptomic landscape of triple-negative breast cancers: subtypes and treatment strategies. Cancer Cell. 2019;35:428-440.e5.

13. Burstein MD, Tsimelzon A, Poage GM, Covington KR, Contreras A, Fuqua SAW, et al. Comprehensive genomic analysis identifies novel subtypes and targets of triple-negative. Clin Cancer Res. 2015;21:1688-98.

14. Liu Y-R, Jiang Y-Z, Xu X-E, Yu K-D, Jin X, Hu X, et al. Comprehensive transcriptome analysis identifies novel molecular subtypes and subtype-specific RNAs of triple-negative breast cancer. Breast Cancer Res. 2016;18:33.

15. Abd El-Rehim DM, Pinder SE, Paish CE, Bell J, Blamey RW, Robertson JFR, et al. Expression of luminal and basal cytokeratins in human breast carcinoma. J Pathol. 2004;203:661-71.

16. Reis-Filho JS, Simpson PT, Martins A, Preto A, Gärtner F, Schmitt FC. Distribution of p63, cytokeratins 5/6 and cytokeratin 14 in 51 normal and 400 neoplastic human tissue samples using TARP-4 multi-tumor tissue microarray. Virchows Arch Int J Pathol. 2003;443:122-32.

17. Koo JS, Jung W. Clinicopathlogic and immunohistochemical characteristics of triple negative invasive lobular carcinoma. Yonsei Med J. 2011;52:89-97.

18. Montagna E, Maisonneuve P, Rotmensz N, Cancello G, Iorfida M, Balduzzi A, et al. Heterogeneity of triple-negative breast cancer: histologic subtyping to inform the outcome. Clin Breast Cancer. 2013;13:31-39.

19. Harbhajanka A, Lamzabi I, Singh RI, Ghai R, Reddy VB, Bitterman P, et al. Correlation of clinicopathologic parameters and immunohistochemical features of triple-negative invasive lobular carcinoma. Appl Immunohistochem Mol Morphol. 2014;22: e18-26.

20. Iorfida M, Maiorano E, Orvieto E, Maisonneuve P, Bottiglieri L, Rotmensz N, et al. Invasive lobular breast cancer: subtypes and outcome. Breast Cancer Res Treat. 2012;133:713-23.

21. Amin MB, Edge S, Greene F, Byrd DR, Brookland RK, Washington MK, et al., editors. AJCC cancer staging manual. 8th ed. Springer International Publishing; 2017, https://www.springer. com/us/book/9783319406176

22. Elston CW, Ellis IO. Pathological prognostic factors in breast cancer. I. The value of histological grade in breast cancer: experience from a large study with long-term follow-up. Histopathology. 1991;19:403-10.

23. Wolff AC, Hammond MEH, Allison KH, Harvey BE, Mangu PB, Bartlett JMS, et al. Human epidermal growth factor receptor 2 testing in breast cancer: American Society of Clinical Oncology/ College of American Pathologists Clinical Practice Guideline Focused Update. Arch Pathol Lab Med. 2018;142:1364-82. 
24. Canas-Marques R, Schnitt SJ. E-cadherin immunohistochemistry in breast pathology: uses and pitfalls. Histopathology. 2016;68:57-69.

25. Da Silva L, Parry S, Reid L, Keith P, Waddell N, Kossai M, et al. Aberrant expression of E-cadherin in lobular carcinomas of the breast. Am J Surg Pathol. 2008;32:773-83.

26. Bonnefoi H, MacGrogan G, Poncet C, Iggo R, Pommeret F, Grellety T, et al. Molecular apocrine tumours in EORTC 10994/ BIG 1-00 phase III study: pathological response after neoadjuvant chemotherapy and clinical outcomes. $\mathrm{Br} \mathrm{J}$ Cancer. 2019;120:913-21.

27. Zhao S, Ma D, Xiao Y, Li X-M, Ma J-L, Zhang H, et al. Molecular subtyping of triple-negative breast cancers by immunohistochemistry: molecular basis and clinical relevance. Oncologist. 2020. https://doi.org/10.1634/theoncologist.2019-0982.

28. Alsadoun N, MacGrogan G, Truntzer C, Lacroix-Triki M, Bedgedjian I, Koeb M-H, et al. Solid papillary carcinoma with reverse polarity of the breast harbors specific morphologic, immunohistochemical and molecular profile in comparison with other benign or malignant papillary lesions of the breast: a comparative study of 9 additional cases. Mod Pathol. 2018;31:1367-80.

29. Réda M, Richard C, Bertaut A, Niogret J, Collot T, Fumet JD, et al. Implementation and use of whole exome sequencing for metastatic solid cancer. EBioMedicine. 2020;51:102624.

30. McLaren W, Gil L, Hunt SE, Riat HS, Ritchie GRS, Thormann A, et al. The ensembl variant effect predictor. Genome Biol. 2016;17:122.

31. Denkert C, Liedtke C, Tutt A, von Minckwitz G. Molecular alterations in triple-negative breast cancer-the road to new treatment strategies. Lancet. 2017;389:2430-42.

32. Desmedt C, Zoppoli G, Sotiriou C, Salgado R. Transcriptomic and genomic features of invasive lobular breast cancer. Semin Cancer Biol. 2017;44:98-105.

33. Bray NL, Pimentel H, Melsted P, Pachter L. Near-optimal probabilistic RNA-seq quantification. Nat Biotechnol 2016;34:525-7.

34. Love MI, Huber W, Anders S. Moderated estimation of fold change and dispersion for RNA-seq data with DESeq2. Genome Biol. 2014;15:550.

35. Parker JS, Mullins M, Cheang MCU, Leung S, Voduc D, Vickery $\mathrm{T}$, et al. Supervised risk predictor of breast cancer based on intrinsic subtypes. J Clin Oncol. 2009;27:1160-7.

36. Gendoo DMA, Ratanasirigulchai N, Schroeder MS, Pare L, Parker JS, Prat A, et al. genefu: computation of gene expression-based signatures in breast cancer. R package version 2.22.0, http://www. pmgenomics.ca/bhklab/software/genefu.

37. Chen X, Li J, Gray WH, Lehmann BD, Bauer JA, Shyr Y, et al. TNBCtype: a subtyping tool for triple-negative breast cancer. Cancer Inform. 2012;11:147-56.

38. Stein RA, Chang C-Y, Kazmin DA, Way J, Schroeder T, Wergin $\mathrm{M}$, et al. Estrogen-related receptor alpha is critical for the growth of estrogen receptor-negative. Breast Cancer Cancer Res. 2008;68:8805-12.

39. Jarzabek K, Koda M, Kozlowski L, Sulkowski S, Kottler M-L, Wolczynski S. The significance of the expression of ERRalpha as a potential biomarker in breast cancer. J Steroid Biochem Mol Biol. 2009;113:127-33.
40. Mills MN, Yang GQ, Oliver DE, Liveringhouse CL, Ahmed KA, Orman AG, et al. Histologic heterogeneity of triple negative breast cancer: a National Cancer Centre Database analysis. Eur J Cancer. 2018;98:48-58.

41. Mayer IA, Abramson VG, Lehmann BD, Pietenpol JA. New strategies for triple-negative breast cancer-deciphering the heterogeneity. Clin Cancer Res. 2014;20:782-90.

42. Schmid P, Adams S, Rugo HS, Schneeweiss A, Barrios CH, Iwata $\mathrm{H}$, et al. Atezolizumab and nab-paclitaxel in advanced triplenegative breast cancer. N Engl J Med. 2018;379:2108-21.

43. Groenendijk FH, Treece T, Yoder E, Baron P, Beitsch P, Audeh $\mathrm{W}$, et al. Estrogen receptor variants in ER-positive basal-type breast cancers responding to therapy like ER-negative breast cancers. NPJ Breast Cancer. 2019;5:15.

44. Bertucci F, Finetti P, Cervera N, Esterni B, Hermitte F, Viens P, et al. How basal are triple-negative breast cancers? Int $\mathrm{J}$ Cancer. 2008;123:236-40.

45. Nielsen TO, Hsu FD, Jensen K, Cheang M, Karaca G, Hu Z, et al. Immunohistochemical and clinical characterization of the basallike subtype of invasive breast carcinoma. Clin Cancer Res. 2004;10:5367-74.

46. Cimino-Mathews A, Subhawong AP, Elwood H, Warzecha HN, Sharma R, Park BH, et al. Neural crest transcription factor Sox 10 is preferentially expressed in triple-negative and metaplastic breast carcinomas. Hum Pathol. 2013;44:959-65.

47. McNamara KM, Yoda T, Takagi K, Miki Y, Suzuki T, Sasano H. Androgen receptor in triple negative breast cancer. J Steroid Biochem Mol Biol. 2013;133:66-76.

48. Robinson JLL, Macarthur S, Ross-Innes CS, Tilley WD, Neal DE, Mills IG, et al. Androgen receptor driven transcription in molecular apocrine breast cancer is mediated by FoxA1. EMBO J. 2011;30:3019-27.

49. Deblois G, Giguère V. Oestrogen-related receptors in breast cancer: control of cellular metabolism and beyond. Nat Rev Cancer. 2013;13:27-36.

50. Deblois G, Hall JA, Perry M-C, Laganière J, Ghahremani M, Park $\mathrm{M}$, et al. Genome-wide identification of direct target genes implicates estrogen-related receptor alpha as a determinant of breast cancer heterogeneity. Cancer Res. 2009;69:6149-57.

51. Teyssier C, Bianco S, Lanvin O, Vanacker J-M. The orphan receptor ERRalpha interferes with steroid signaling. Nucleic Acids Res. 2008;36:5350-61.

52. Manna S, Bostner J, Sun Y, Miller LD, Alayev A, Schwartz NS, et al. ERR $\alpha$ is a marker of tamoxifen response and survival in triple-negative breast cancer. Clin Cancer Res. 2016;22:1421-31.

53. Gerratana L, Basile D, Buono G, De Placido S, Giuliano M, Minichillo $S$, et al. Androgen receptor in triple negative breast cancer: a potential target for the targetless subtype. Cancer Treat Rev. 2018;68:102-10.

54. Bonnefoi H, Grellety T, Tredan O, Saghatchian M, Dalenc F, Mailliez A, et al. A phase II trial of abiraterone acetate plus prednisone in patients with triple-negative androgen receptor positive locally advanced or metastatic breast cancer (UCBG 12-1). Ann Oncol. 2016;27:812-8.

\title{
Affiliations
}

\author{
Anthony Bergeron $\mathbb{1}^{1} \cdot$ Gaëtan MacGrogan $\mathbb{1}^{2,3} \cdot$ Aurélie Bertaut ${ }^{4}$ Sylvain Ladoire $5,6,7$ Patrick Arveux ${ }^{8}$. \\ Isabelle Desmoulins ${ }^{5}$ - Hervé Bonnefoi ${ }^{3,9,10}$ - Catherine Loustalot $^{11}$. Sophie Auriol ${ }^{12}$. Françoise Beltjens ${ }^{1}$. \\ Emilie Degrolard-Courcet ${ }^{1}$ - Céline Charon-Barra ${ }^{1} \cdot$ Corentin Richard ${ }^{13} \cdot$ Romain Boidot $\mathbb{D}^{13} \cdot$ Laurent Arnould $\mathbb{D}^{1,6}$
}


1 Unit of Pathology, Department of Biology and Pathology of the Tumors, Centre Georges-François Leclerc, Dijon, France

2 Department of Biopathology, Institut Bergonié, Bordeaux, France

3 INSERM U1218, Bordeaux, France

4 Unit of Methodology and Biostatistics, Centre Georges-François Leclerc, Dijon, France

5 Department of Medical Oncology, Centre Georges-François Leclerc, Dijon, France

6 INSERM U1231, Dijon, France

7 University of Burgundy-Franche Comté, Dijon, France
8 Department of Epidemiology, Centre Georges-François Leclerc, Dijon, France

9 Department of Medical Oncology, Institut Bergonié, Bordeaux, France

10 University of Bordeaux, Bordeaux, France

11 Department of Surgery, Centre Georges-François Leclerc, Dijon, France

12 Department of Surgery, Institut Bergonié, Bordeaux, France

13 Unit of Molecular Pathology, Department of Biology and Pathology of the Tumors, Centre Georges-François Leclerc, Dijon, France 